\title{
Health related quality of life in patients with multiple myeloma undergoing a double transplantation
}

Uyl-de Groot CA, Buijt I, Gloudemans IJM, Ossenkoppele GJ, van den Berg HP, Huijgens PC. Health related quality of life in patients with multiple myeloma undergoing a double transplantation.

Eur J Haematol 2005: 74: 136-143. (c) Blackwell Munksgaard 2005.

Abstract: Objectives: To investigate the subjective well-being of patients with newly diagnosed multiple myeloma who were treated in a tandem transplantation programme. Methods: Fifty-one patients participated in the prospective, longitudinal questionnaire study. The EORTC QLQ-C30 and the EuroQol-5D were administered 2 wk after completion of vincristine, adriamycin and dexamethason/vincristine, adriamycin and methyl prednison (VAD/VAMP) chemotherapy, both at hospital discharge after treatment with high-dose melphalan (HDM) and 1 month after this hospital discharge, at hospital admission, at the day of hospital discharge for peripheral stem cell transplantation (PSCT) and at 6 and 12 months following discharge after

PSCT. Results: Overall, patients' functioning improved during treatment and follow-up, with significant decreases shortly following PSCT. Shortly after HDM and PSCT, patients reported a considerable increase in levels of soreness in the mouth $(+26 /+36$ points on a scale ranging form 0 to 100; $P<0.01)$, change of taste $(+23 /+21$ points; $P<0.05 /$ NS), nausea/vomiting $(+26 /+27$ points; $P<0.01 /<0.05)$, appetite loss $(+40 /+43$ points; $P<0.001)$ and diarrhoea $(+25 /+36$ points; $P<0.01)$. However, none of these symptoms persisted during followup. Conclusion: The intensive treatment programme was subjectively being well tolerated by the majority of patients. The duration of declined quality of life after administration of HDM seemed to be short. The duration of subjective recovery after PSCT remained uncertain, but in any case was present at the 6 month follow-up. Together with the rather good results in survival, the evaluation of quality of life invites further exploration of double transplantations in multiple myeloma.
C. A. Uyl-de Groot ${ }^{1}$, I. Buijt ${ }^{1}$, I. J. M. Gloudemans $^{2}$, G. J. Ossenkoppele ${ }^{2}$, H. P. van den Berg ${ }^{2}$, P. C. Huijgens ${ }^{2}$

${ }^{1}$ Institute for Medical Technology Assessment, Erasmus Medical Centre, Rotterdam, The Netherlands; ${ }^{2}$ VU University Medical Centre, Amsterdam, The Netherlands

Key words: multiple myeloma; quality of life; tandem transplantations; EORTC OLQ-C30; EuroOol-5D

Correspondence: Carin A. Uyl-de Groot, iMTA, Erasmus MC, Erasmus Medical Centre Rotterdam, P0 Box 1738, 3000 DR Rotterdam, The Netherlands Fax: + 310104089081 e-mail: Uyl@bmg.eur.nl

Accepted for publication 2 September 2004
Multiple myeloma is a diffuse neoplasm of bone marrow plasma cells in which the malignant cells mingle with normal haematopoietic cells throughout the red bone marrow. Most frequent complications of multiple myeloma are painful pathologic fractures, anaemia, hypocalcaemia, renal failure and recurrent bacterial infections (1-3). Obviously, the subjective experience of numerous symptoms caused by these complications reduces the ability of normal functioning and negatively influences patients' quality of life.

Despite intensive research for several decades, cure is not presently attainable with standard chemotherapy, interferon or even with high-dose chemotherapy supported by autologous transplantation regimens with bone marrow or peripheral blood stem cells (4-5). Only a small number of patients, for whom a human leucocyte antigen (HLA) identical sibling donor is available, reach long-term remission and, apparently, cure after allogeneic grafting. Thus multiple myeloma is uniformly lethal with a median survival of $<4 \mathrm{yr}$ in most studies (5-7). High-dose therapy with stem cell support results in high rates of response, but in general responses are not long lasting (8-9) and survival plateaus do not appear (10). Because of the poor prognosis for long-term (disease-free) survival in myeloma patients and the fact that there are no 
major survival differences between existing treatments, quality of life should be an important consideration in the choice of treatment.

In an attempt to improve prognosis in myeloma, several studies are now being performed using 'total therapy' as an approach. This involves a strategy of two (or more) high-dose therapy and rescue procedures (11-13). In the VU University Medical Centre, Amsterdam, such a double-transplant treatment programme has been applied for several years. We explore a protocol using vincristine, adriamycin and dexamethason (VAD) induction therapy, followed by high-dose melphalan (HDM) $\left(140 \mathrm{mg} / \mathrm{m}^{2}\right)$ and whole blood stem cells. Thereafter, stem cells are harvested by leucapheresis and a second stem cell transplantation is performed after busulphan $(16 \mathrm{mg} / \mathrm{kg})$ and cyclophosphamide $(120 \mathrm{mg} / \mathrm{kg})$. For those patients who progress to the second transplantation, the median overall survival is 84 months (range 14-152), rated from the time of diagnosis (14).

The aim of the current quality of life study was to evaluate the subjective well-being of patients participating in this intensive double transplantation programme.

\section{Methods}

Study population

All patients scheduled for intensive treatment of multiple myeloma in the VU University Medical Centre, Amsterdam, the Netherlands between March 1997 and December 1998 were requested to participate in the quality of life study. In addition, the collaboration was asked of patients who already started treatment, but who had not yet passed the last two measurement points for quality of life. The total study population for quality of life consisted of 51 patients: 35 patients from the start of the protocol and 16 patients who took in later. These latter patients all started treatment between March 1995 and September 1996.

All patients referred to the hospital who were $<65 \mathrm{yr}$ of age with or without prior treatment for multiple myeloma were eligible for the intensive treatment programme. Exclusion criteria were severely abnormal cardiac or pulmonary function, serum creatinin above $250 \mu \mathrm{mol} / \mathrm{L}$, serum bilirubin above $35 \mu \mathrm{mol} / \mathrm{L}$, alanine-aminotransferase (ALAT) and/or aspartate-aminotransferase (ASAT) above four times normal, other severe non-haematological disease, or refusal to participate.

All patients were scheduled for the following treatment protocol: two courses of VAD or vincristine, adriamycin and methyl prednison (VAMP) chemotherapy, HDM followed by transplantation of whole blood, collection of r-met Hu G-CSF mobilised peripheral blood progenitor cells by leukapheresis and finally high-dose chemotherapy (busulfan/cyclophosphamide) followed by reinfusion of the previously collected peripheral stem cells (PSCT) (14).

The VU University Medical Centre is an academic hospital and the haematologists have a consultation function for several local hospitals. The policy of the haematologists is to treat referred patients in the academic hospital when needed, but in the referring hospital when possible. Because VAD/VAMP chemotherapy can be given safely in a non-academic hospital, most patients started their treatment in a local hospital.

\section{The questionnaire}

Both the EORTC QLQ-C30 and the EuroQol 5-D were used in this study. The EORTC QLQ-C30 (15) is a cancer-specific questionnaire. It consists of five functional scales (physical, role, emotional, cognitive and social functioning), three symptom scales (fatigue, pain and nausea/vomiting), six single items (dyspnoea, appetite loss, sleep disturbance, constipation, diarrhoea and financial impact of disease) and one global health and quality of life scale. All scores can be linearly transformed into a scale ranging from 0 to 100 . Higher scores on the functioning scales represent higher levels of functioning, whereas higher scores on the symptom scales represent a higher level of complaints. The questionnaire is available in many languages and its reliability and validity in myeloma patients has been proven in a large Scandinavian study (16). At the time the current study started, there was not yet a myeloma specific module of the EORTC questionnaire available. Therefore, some disease and treatment specific items were added by the researchers. These items were formulated in collaboration with haematologists and tested on a limited number of patients for clarity and relevance in another study in myeloma patients performed by the institute for Medical Technology Assessment (iMTA). The items are presented in Appendix and had the same format as the items of the EORTC QLQ-C30.

The EuroQol-5D was included in this study because of its ability to express health related quality of life in one summarised number, which can be used as a utility value in economic evaluations. It is a generic instrument, which measures five dimensions: mobility, self-care, usual activities, pain/discomfort and anxiety/depression (17). In the descriptive part the patient is required to rate his or her health on each of the five dimensions by checking one of the three levels of severity: no problems, some/moderate problems or severe problems. The EQ-5D Index assigns a preference 


\section{Uyl-de Groot et al.}

value (utility) to each health state generated by the descriptive part of the questionnaire. The preference weights used in this study have been obtained from a sample of the general population in the UK by using the time trade-off procedure (18).

\section{Data collection}

Seven time points for measurement of quality of life were defined:

1 baseline $=2 \mathrm{wk}$ after completion of $\mathrm{VAD} /$ VAMP therapy;

2 the day of hospital discharge after HDM;

31 month after hospital discharge following HDM;

4 the day of hospital admission for PSCT;

5 the day of hospital discharge following PSCT;

66 months after hospital discharge following PSCT;

712 months after hospital discharge following PSCT.

Unfortunately, the fact that most patients started their treatment in a diversity of local hospitals made a pretreatment measurement too complicated. The questionnaires were either handed to the patients on the hospital ward in case they were hospitalised or mailed to the patients' home. Reminders were sent when questionnaires were not returned within 1 month.

\section{Statistical methods}

The paired-samples $t$-test was used to examine whether there were significant changes in quality of life during treatment and follow-up, compared with baseline values. Thus, the main analyses were only based on the patients who completed the baseline questionnaire. To investigate whether the QoL of these patients differed from the patients who failed to complete the baseline questionnaire, absolute scores of both groups on all the other time-points were compared by using the student's $t$-test.

Differences of 10 points or more on the $0-100$ scale were considered to be clinically relevant (19, 20). Changes in scores of $<10$ points were supposed not to be relevant and therefore not statistically tested. The statistical software SPSS for windows version 10.0 was used.

\section{Results}

Of the 36 patients who entered the treatment programme between March 1997 and December 1998, one refused to take part in the quality of life study. This patient found the completion of the questionnaires too much a burden.
The mean age of the participants at the start of treatment was $53 \mathrm{yr}$ (median 54; range 31-65). Patient characteristics and remission status after treatment are shown in Table 1.

The mean duration of the whole treatment programme from start VAD/VAMP until hospital discharge after PSCT for patients who completed the whole programme was about 12 months. Eleven patients never proceeded to PSCT because of disease progression $(n=4)$, insufficient recovery after $\operatorname{HDM}(n=1)$, patient refusal $(n=2)$, anticoagulant therapy $(n=1)$ or insufficient harvest of stem cells $(n=3)$.

\section{Response to questionnaires}

A total of 205 questionnaires was completed. The response rate varied from $71 \%$ after VAD/VAMP to $98 \%$ at hospital discharge following HDM. The number of completed questionnaires at each point in time is shown in Table 2.

The response rate was calculated as the proportion of completed questionnaires of the total number that had to be filled out at each timepoint. Reasons for non-applicability were:

- patient had already passed this time point when he/she entered the study: $n=16$ at T1, $n=15$ at $\mathrm{T} 2, n=11$ at T3, $n=8$ at T4, $n=7$ at T5;

- patient died: $n=1$ at T5, $n=2$ at T6, $n=5$ at $\mathrm{T} 7 ;$

- patient did not proceed to PSCT: $n=11$ at T4, T5, T6 and T7.

Reasons for missing questionnaires were logistical problems for the measurement after VAD/VAMP (because of treatment in local hospitals), questionnaires were not handed to the patient at the hospital ward $(n=15)$ or refusal by the patient $(n=18)$.

An overview of the available quality of life questionnaires and patterns of missing data is given in Table 3.

From Table 3 it can be read that the number of patients who could be included in the main analysis (change scores from baseline) declines from 24 1 month after hospital discharge for HDM to 12 at 12 months following discharge after PSCT. These small numbers of patients reduce the likeliness of statistical significance, even for relatively large differences in change scores.

\section{The EORTC OLQ-C30}

High-dose melphalan did not seem to have a major impact on patients' functioning scores in the shortterm. At the time of hospital discharge (at a median of $17 \mathrm{~d}$ after administration of HDM) the level of 
Quality of life in multiple myeloma

Table 1. Patient characteristics and response to treatment

\begin{tabular}{|c|c|c|}
\hline & $\begin{array}{l}\text { Patients in main analysis } \\
\qquad(n=25)\end{array}$ & $\begin{array}{l}\text { All patients } \\
(n=51)\end{array}$ \\
\hline \multicolumn{3}{|l|}{ Age: } \\
\hline Mean (SD) & $53(8.2)$ & $53(7.2)$ \\
\hline Median (min/max) & $55(31 / 65)$ & $54(31 / 65)$ \\
\hline \multicolumn{3}{|l|}{$\operatorname{Sex}[n(\%)]$} \\
\hline Male & $16(64)$ & $31(61)$ \\
\hline Female & $9(36)$ & $20(39)$ \\
\hline \multicolumn{3}{|c|}{ Stage Salmon and Durie [ $n(\%)]$} \\
\hline la & $8(32)$ & $12(24)$ \\
\hline Ila & $1(4)$ & $4(8)$ \\
\hline Illa & $15(60)$ & $32(63$ \\
\hline Illb & $1(4)$ & $3(6)$ \\
\hline \multicolumn{3}{|c|}{ Remission status after $\mathrm{HDM}^{1}[n(\%)]$} \\
\hline Complete remission & $7(28)$ & $13(25)$ \\
\hline Partial remission & $8(32)$ & $16(31)$ \\
\hline Improvement & $1(4)$ & $7(14)$ \\
\hline No response & $8(32)$ & $13(25)$ \\
\hline Toxic death & $1(4)$ & $1(2)$ \\
\hline Unknown & & $1(2)$ \\
\hline \multicolumn{3}{|c|}{ Remission status after $\mathrm{PSCT}^{1}[n(\%)]$} \\
\hline Complete remission & $5(20)$ & $11(22)$ \\
\hline Partial remission & $8(32)$ & $15(29)$ \\
\hline Improvement & $2(8)$ & $8(16)$ \\
\hline No response & $2(8)$ & $3(6)$ \\
\hline Toxic death & & $1(2)$ \\
\hline
\end{tabular}

${ }^{1}$ Complete remission, complete disappearance of monoclonal proteins on immunofixation, $<5 \%$ plasma cells in the bone marrow and stable skeletal lesions; partial remission, $>75 \%$ reduction in monoclonal proteins; improvement, a reduction between $50 \%$ and $75 \%$. No response represents a reduction of $<50 \%$. Toxic death, death within 3 months of transplantation.

functioning was comparable to that at baseline. One month after discharge, even a significant improvement in physical $(P<0.01)$, role $(P<0.05)$ and social $(P<0.05)$ functioning was reported. At the time of admission for PSCT (at a median time of 9 months after HDM) a further improvement in global quality of life $(P<0.05)$ was seen. As could be expected, an enormous decline in the level of functioning was observed by the time of discharge following PSCT. At 6 and 12 months after PSCT, physical, role and social functioning were higher than at baseline.

Regarding the symptoms measured by the QLQC30, there was a considerable increase in levels of appetite loss $(P<0.01)$, diarrhoea $(P<0.01)$ and nausea/vomiting $(P<0.01)$ after the administration of HDM and after PSCT. These symptoms did

Table 2. Response to questionnaires by measurement

\begin{tabular}{lcrrrrrr}
\hline & T1 & T2 & T3 & T4 & T5 & T6 & T7 \\
\hline Completed & 25 & 31 & 39 & 27 & 26 & 31 & 26 \\
Missing & 10 & 5 & 1 & 5 & 5 & 7 & 9 \\
Not applicable & 16 & 15 & 11 & 19 & 19 & 13 & 16 \\
Total & 51 & 51 & 51 & 51 & 51 & 51 & 51 \\
Response rate & 0.71 & 0.86 & 0.98 & 0.84 & 0.84 & 0.82 & 0.74 \\
\hline
\end{tabular}

T1, after VAD/NAMP; T2, discharge HDM; T3, 1 month after HDM; T4, admission PBSCT; T5, discharge PBSCT; T6, 6 months follow-up; T7, 12 months follow-up.
Table 3. Available quality of life questionnaires and patterns of missing data

\begin{tabular}{cccccccc}
\hline T1 & T2 & T3 & T4 & T5 & T6 & T7 & Frequency \\
\hline$*$ & $*$ & $*$ & $*$ & $*$ & $*$ & $*$ & 10 \\
$*$ & $*$ & $*$ & $*$ & $*$ & $*$ & & 3 \\
$*$ & $*$ & $*$ & & & & & 9 \\
$*$ & & $*$ & $*$ & & $*$ & $*$ & 1 \\
$*$ & & $*$ & & & & & 1 \\
$*$ & & & $*$ & $*$ & $*$ & $*$ & 1 \\
& $*$ & $*$ & $*$ & $*$ & $*$ & $*$ & 2 \\
& $*$ & $*$ & $*$ & $*$ & & & 1 \\
$*$ & $*$ & $*$ & & & & 2 \\
$*$ & $*$ & & & & & 4 \\
& $*$ & $*$ & $*$ & $*$ & $*$ & 1 \\
& $*$ & $*$ & $*$ & $*$ & & 2 \\
& $*$ & $*$ & $*$ & & & 1 \\
& $*$ & & $*$ & & & 1 \\
& $*$ & & & & & 1 \\
& & $*$ & $*$ & $*$ & $*$ & 3 \\
& & & $*$ & $*$ & $*$ & 1 \\
& & & & $*$ & $*$ & 6 \\
& & & & $*$ & & 1
\end{tabular}

T1, baseline (after VAD/NAMP); T2, discharge HDM; T3, 1 month after HDM; T4, admission PBSCT; T5, discharge PBSCT; T6, 6 months follow-up; T7, 12 months follow-up.

not persist during follow-up. Mean change scores from baseline for selected variables are presented in Table 4.

Treatment and disease specific items

After VAD/VAMP, patients complained most about diminished sexual interest (mean absolute score $=52$ ), pain in the back (mean absolute score $=43$ ) and bone pain (mean absolute score $=35$ ). Although diminished sexual interest showed a mean decrease of 22 points (NS) at 12 months follow-up, the problem remained present in many patients.

Once patients had received HDM, the level of bone pain declined significantly $(P<0.05)$. However, this decline did not remain to this extent during further treatment and follow-up. Pain in the back also showed a decline, but the mean change score was only significant shortly after HDM and PSCT and the mean absolute score was still 32 after 12 months of follow-up.

The intensity of soreness in the mouth $(P<0.01)$ and change of taste $(P<0.05)$ increased after administration of HDM and after PSCT, but most patients were no longer bothered by these symptoms at 1 month after HDM and at 6 months after PBCT. Mean change scores for selected symptoms are shown in Table 4 and in Fig. 1.

\section{EuroQol utilities}

At baseline, the combination of problems on the five EuroQol dimensions correspond to a mean 


\begin{tabular}{|c|c|c|c|c|c|c|c|}
\hline & $\begin{array}{l}\text { Baseline } \\
(n=25)\end{array}$ & $\begin{array}{c}\text { T2 } \\
(n=22)\end{array}$ & $\begin{array}{c}\text { T3 } \\
(n=24)\end{array}$ & $\begin{array}{c}\text { T4 } \\
(n=15)\end{array}$ & $\begin{array}{c}\text { T5 } \\
(n=14)\end{array}$ & $\begin{array}{c}\text { T6 } \\
(n=15)\end{array}$ & $\begin{array}{c}\mathrm{T7} \\
(n=12)\end{array}$ \\
\hline \multicolumn{8}{|c|}{ EORTC OLQ-C30 functioning scales } \\
\hline Physical functioning & $50(28)$ & -2 & $13^{* *}$ & $13^{*}$ & $-19^{*}$ & $13^{*}$ & $20^{*}$ \\
\hline Role functioning & $41(29)$ & 2 & $18^{* *}$ & 14 & $-26^{* *}$ & $19^{*}$ & 20 \\
\hline Emotional functioning & $72(22)$ & 3 & $10^{*}$ & 6 & 0 & 0 & 1 \\
\hline Cognitive functioning & $76(25)$ & -11 & 8 & 1 & -6 & 3 & 3 \\
\hline Social functioning & $59(30)$ & 5 & 12 & 6 & $-23^{*}$ & 10 & $13^{*}$ \\
\hline Global QoL & $58(23)$ & $-11^{*}$ & 3 & $10^{*}$ & $-17^{* *}$ & 7 & 4 \\
\hline \multicolumn{8}{|l|}{ EORTC OLQ-C30 symptoms } \\
\hline Fatigue & $55(29)$ & 7 & $-15^{*}$ & -13 & 10 & -13 & -6 \\
\hline Nausea/vomiting & $11(25)$ & $26^{* *}$ & 2 & -1 & $27^{*}$ & -1 & 4 \\
\hline Pain & $37(29)$ & -7 & -8 & -10 & 4 & -9 & -11 \\
\hline Appetite loss & $22(31)$ & $40^{* *}$ & 2 & -4 & $43^{* *}$ & -4 & -3 \\
\hline Diarrhoea & $18(31)$ & $25^{* *}$ & -1 & 0 & $36^{* *}$ & -2 & 3 \\
\hline \multicolumn{8}{|c|}{ Disease/treatment related symptoms } \\
\hline Pain in the back & $43(37)$ & -6 & $-14^{*}$ & -7 & $-21^{*}$ & -7 & -11 \\
\hline Soreness of the mouth & $9(20)$ & $26^{* *}$ & 1 & -11 & $36^{* *}$ & -2 & -6 \\
\hline Change in taste & $20(32)$ & $23^{*}$ & 6 & -9 & 21 & -4 & -8 \\
\hline Diminished sexual interest & $52(40)$ & 11 & -1 & $-27^{*}$ & -12 & -20 & -22 \\
\hline Pain in the bones & $35(35)$ & $-20^{*}$ & -4 & -7 & $-21^{*}$ & -9 & -6 \\
\hline Euro0ol utility & $0.52(0.33)$ & 0.03 & $0.14^{*}$ & 0.14 & -0.14 & 0.12 & 0.17 \\
\hline
\end{tabular}

${ }^{*} P<0.05 ;{ }^{* *} P<0.01$.

For the functioning scales and the Euro0ol a negative change score means a deterioration in functioning/utility, whereas for the symptoms a negative change score reflects a lower level of symptoms.

T2, discharge HDM; T3, 1 month after HDM; T4, admission PSCT; T5, discharge PSCT; T6, 6 months follow-up; T7,

12 months follow-up

health utility index of 0.52 on a scale ranging from 0 (death) to 1 (full health). An increase in mean utility is observed from the time point 1 month after discharge onwards, with a temporary decrease at hospital discharge after PSCT and a maximum increase of 0.17 at 12 months follow-up $(P=0.06)$.

\section{Absolute QoL values}

To check whether there were differences in QoL between patients who completed the baseline questionnaire and the other patients, their mean absolute scores on all dimensions and symptoms were compared at every time-point. No relevant/significant differences were observed.

However, absolute scores of patients at 6 and 12 months follow-up were better than is being suggested by mean change scores from baseline in Table 4. For example, the mean absolute score at 12 months follow-up for physical functioning is 85 , whereas a mean score of 70 is suggested (mean baseline score is 50, mean change score from baseline $=20$ ). The absolute scores at baseline and 12 months follow-up are shown in Table 5.

\section{Discussion}

In our quality of life study, we found an improvement in subjective well-being for patients who were able to complete the treatment programme. This improvement was not only found in analysing the cancer specific EORTC QLQ-C30 questionnaire
Table 4. Mean absolute scores (SD) at baseline after VAD/VAMP (baseline) and mean change scores from baseline and myeloma specific symptoms, but also in (generic) EuroQol-5D utilities.

This was a single centre, non-randomised study, so patient selection cannot be excluded. However, the advantage of such data is that they reflect real clinical practice. The number of patients in the main analyses was small. This was because only patients with a baseline assessment could be included to calculate change scores from baseline. The fact that mean absolute scores on all the other timepoints did not differ between patients with or without a baseline measurement, suggests that the results are also valid for the whole study population of 51 patients. The patient numbers in the analyses were further reduced by missing questionnaires. If non-response to questionnaires is selective, the results might be under- or overestimated. We have no reasons to assume that the non-response to questionnaires in our study was selective. Reasons for missing questionnaires were not always known. In any case, response to questionnaires was not related to objective disease state (remission or progressive disease). So, selection of patients in better clinical condition seems unlikely.

Unfortunately, our baseline measurement was not a pretreatment assessment. Because of logistic problems, a pretreatment measurement was not possible. Because VAD/VAMP chemotherapy is known for its rapid tumour reduction (21), it can be assumed that pretreatment levels of quality of life were lower compared with our baseline measurement after VAD/VAMP. This means that the real 

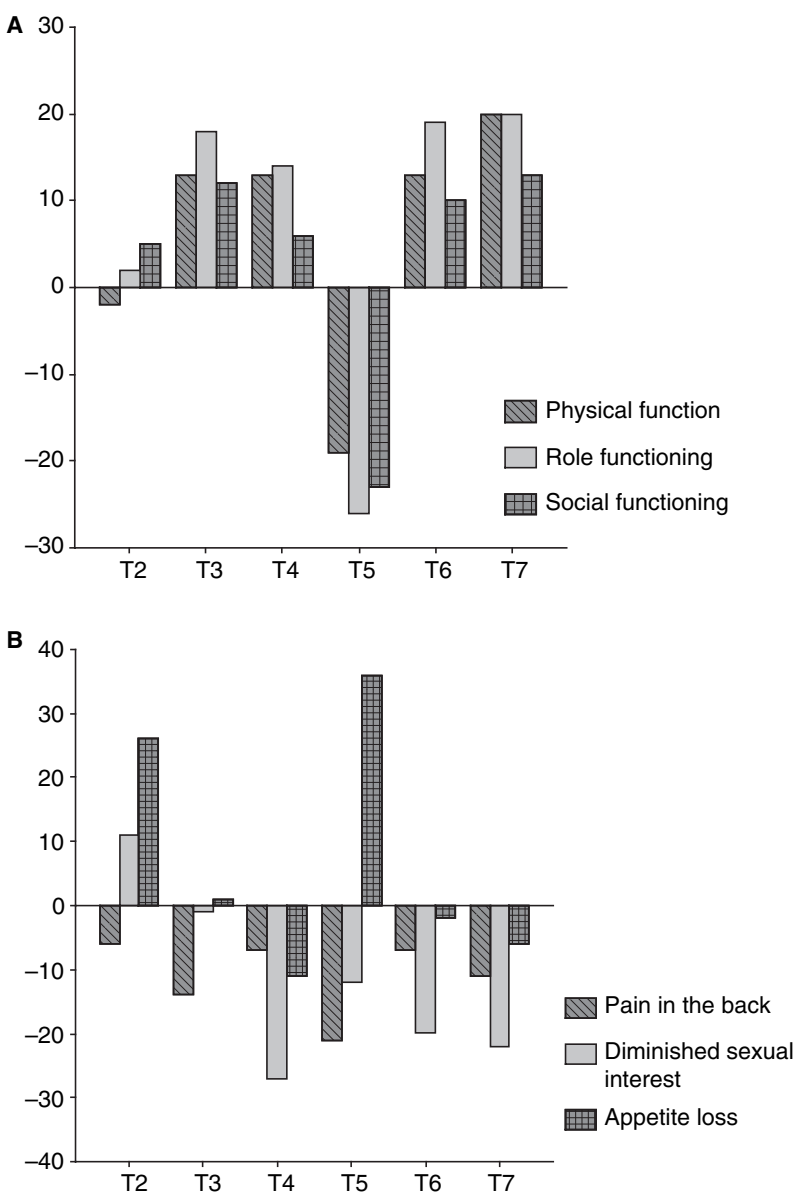

Fig. 1. (A) Mean change scores for preselected functional dimensions. (B) Mean change scores for preselected symptoms. T2, discharge HDM; T3, 1 month after HDM; T4, admission PSCT; T5, discharge PSCT; T6, 6 months followup; T7, 12 months follow-up. improvement in quality of life during therapy is likely to be underestimated in our study.

As expected, the largest decline in quality of life was observed at the time of hospital discharge following HDM and PSCT. These declines were obviously treatment related. Patients reported improvements at 1 month after HDM and at 6 months after PSCT. Because there were no measurements between discharge and 6 months after discharge following PSCT, more precise statements about the time to subjective recovery after PSCT cannot be made.

With low mean absolute scores at baseline and small mean change scores during treatment and follow-up, many of the disease and treatment specific symptoms we added to the questionnaire were not relevant; at least, not at the time points of measurement. By now, an official EORTC myeloma module has been developed and is being validated in a number of international studies (22). Not surprisingly, this module has some overlapping with the items we selected.

It is difficult to compare our results with other studies. There is a lack of longitudinal studies in this patient group. In a Scandinavian study (also nonrandomised) quality of life was measured before and after high-dose melpalan with autologous blood stem-cell support (23). Although different from our patients (only one transplantation and analysis on intention to treat basis where $78 \%$ of patients actually proceeded to HDM), the absolute EORTC QLQ-C30 scores of 12 months after start of induction therapy with VAD courses, which corresponds to a mean of 7.5 months after HDM, are comparable

Table 5. Mean absolute scores (SD) at baseline and at 12 months follow-up for the patients who proceeded to PSCT and 12 months follow-up

\begin{tabular}{|c|c|c|c|}
\hline & $\begin{array}{l}\text { Baseline - Patients who } \\
\text { proceeded to } 12 \text { months } \\
\quad \text { follow-up ( } n=12 \text { ) }\end{array}$ & $\begin{array}{l}12 \text { months follow-up - } \\
\text { Patients with baseline } \\
\quad(n=12)\end{array}$ & $\begin{array}{l}12 \text { months follow-up } \\
\text { All patients }(n=26)\end{array}$ \\
\hline \multicolumn{4}{|c|}{ EORTC OLQ-C30 functioning scales } \\
\hline Physical functioning & $65(28)$ & $85(15)$ & $78(19)$ \\
\hline Role functioning & $53(32)$ & $72(18)$ & $71(21)$ \\
\hline Emotional functioning & 74 (19) & $74(20)$ & 78 (19) \\
\hline Cognitive functioning & $79(21)$ & $82(21)$ & 85 (17) \\
\hline Social functioning & $69(27)$ & $82(25)$ & $82(25)$ \\
\hline Global QoL & $66(23)$ & $70(16)$ & 69 (19) \\
\hline \multicolumn{4}{|l|}{ EORTC OLQ-C30 symptoms } \\
\hline Fatigue & $42(30)$ & $35(27)$ & $30(26)$ \\
\hline Nausea/vomiting & $1(5)$ & $6(13)$ & $3(9)$ \\
\hline Pain & $28(30)$ & $17(17)$ & $15(16)$ \\
\hline Appetite loss & $6(13)$ & $3(10)$ & $1(7)$ \\
\hline Diarrhoea & $0(0)$ & $3(10)$ & $1(7)$ \\
\hline \multicolumn{4}{|c|}{ Disease/treatment related symptoms } \\
\hline Pain in the back & 31 (39) & 19 (17) & $22(19)$ \\
\hline Soreness of the mouth & $11(22)$ & $6(13)$ & $4(11)$ \\
\hline Change in taste & $14(22)$ & $6(13)$ & $7(22)$ \\
\hline Diminished sexual interest & $56(38)$ & $33(40)$ & $40(38)$ \\
\hline Pain in the bones & $19(30)$ & $14(17)$ & $17(7)$ \\
\hline Euro0ol utility & $0.60(0.33)$ & $0.77(0.13)$ & $0.79(0.18)$ \\
\hline
\end{tabular}

For the functioning scales and the Euro0ol a negative change score means a deterioration in functioning/utility, whereas for the symptoms a negative change score reflects a lower level of symptoms. 
with the absolute scores of our patients at 6 months after PSCT. In addition, the EORTC scores of our heavily treated patient group were not worse than those of myeloma patients in a large Nordic study who were treated with oral melphalan/prednison (16). This is known as a much less toxic therapy, but the patients were of a higher age (median 67).

Because our time points for measurement of quality of life were related to the actual treatment, we were able to gain insight into the toxicity of the various phases of treatment. In a comparative trial with analysis on intention to treat basis, fixed time points from start of treatment might be preferable, but in our explorative study the choice for treatment related time points seems valid.

Absolute scores of patients at 6 and 12 months follow-up were better than is being suggested by mean scores. This is observed in several dimensions and finds its explanation in the fact that patients who did not proceed to PSCT and 12 monts had poorer quality of life at baseline, This may imply that quality of life at diagnosis might have a prognostic value for treatment outcome. The association between baseline quality of life values and survival has already been described by Wisloff and Hjorth (24). The number of patients in our study was too small for detailed analyses, but this topic might be interesting for future studies.

We conclude that patients who are able to complete the programme show a considerable improvement in quality of life. Because of small patient numbers many of the differences are not statistically significant, but the trend towards improved functioning and reduced symptoms is obvious. This means that the intensive treatment programme is subjectively being tolerated well in the majority of patients. Together with the rather good results in survival, the evaluation of quality of life invites to further exploring double transplantations in multiple myeloma.

\section{Acknowledgement}

This study was supported by a grant from Amgen, The Netherlands.

\section{References}

1. Maclennan ICM, Drayson M, Dunn J. Multiple myeloma. BMJ 1994;308:1033-1036.

2. Alexanian R, Dimopolous MA. The treatment of multiple myeloma. N Engl J Med 1994;330:484-489.

3. Hussein M. Multiple myeloma: an overview of diagnosis and management. Cleve Clin J Med 1994;61:285-298.

4. OKEN MM. Management of multiple myeloma: current and future approaches. Cancer Control 1998;5:218-225.

5. Child JA, Morgan GJ, Davies FE, et al. High-dose chemotherapy with hematopoietic stem-cell rescue for multiple myeloma. N Engl J Med 2003;348:1875-1883.
6. Bersagel DE. Melphalan/prednison vs drug combinations for plasma cell myeloma. Eur J Haematol 1989;43(Suppl. 51): $117-123$.

7. Gregory WM, Richards MA, Malpas JS. Combination of chemotherapy vs melphalan/prednison in the treatment of multiple myeloma: an overview of published trials. J Clin Oncol 1992;10:334-342.

8. Barlogie B, Hall R, Zander A, et al. High-dose melphalan with autologous bone marrow transplantation for multiple myeloma. Blood 1986;67:1298-1301.

9. Cunningham D, Paz-Ares L, Milan S, et al. High-dose melphalan and autologous bone marrow transplantation as consolidation in previously untreated myeloma. J Clin Oncol 1994;12:759-783.

10. Attal M, Harousseau JL, Stoppa AM, et al. A prospective randomized trial of autologous bone marrow transplantation and chemotherapy in multiple myeloma. $\mathrm{N}$ Engl J Med 1996;335:91-97.

11. Barlogie B, Jagannath S, Vesole DH, et al. Superiority of tandem autologous transplantation over standard therapy for previously untreated multiple myeloma. Blood 1997;89:789-793.

12. Attal M, Harousseay JL, Facon T, et al. Single versus double autologous stem-cell transplantation for multiple myeloma. N Engl J Med 2004;349:2495-2502.

13. Harousseau JL, Milpied N, Laporte JP, et al. Double intensive therapy in high risk multiple myeloma. Blood 1992;79:2827-2833.

14. Huijgens PC, Dekker-Roessel van HM, Jonkhoff AR, et al. High-dose melphalan with G-CSF stimulated whole blood rescue followed by stem cell harvesting and busulphan/cyclophosphamide with autologous stem cell transplantation in multiple myeloma. Bone Marrow Transplant 2001;27:925-931.

15. Aaronson NK, Ahmedzai S, Bergman B, et al. The European Organization for Research and Treatment of Cancer QLQ-C30: a quality-of-life instrument for use in international clinical trials in oncology. J Natl Cancer Inst 1993;85:365-376.

16. Wisloff F, Eika S, HipPe E, et al. Measurement of healthrelated quality of life in multiple myeloma. Br J Haematol 1996;92:604-613.

17. The EuroQol Group. EuroQol: a new facility for the measurement of health related quality of life. Health Policy 1990;16:199-208.

18. Dolan, P. Modeling valuations for EuroQol health states. Med Care 1997;35:1095-1108.

19. KING, MT. The interpretation of scores from the EORTC quality of life questionnaires QLQ-C30. Qual Life Res 1996;5:555-567.

20. Osaba D, Rodrigues G, Myles J, et al. Interpreting the significance of changes in health-related quality of life scores. J Clin Oncol 1998;16:139-144.

21. Segeren CM, Sonneveld P, van der Holt B, et al. Vincristine, doxorubicin and dexamethasone (VAD) administered as rapid intravenous infusion for first-line treatment in untreated multiple myeloma. $\mathrm{Br} \mathbf{J}$ Haematol 1999; 105:127-130.

22. Stead ML, Brown JM, Velikova G, et al. Development of an EORTC questionnaire module to be used in healthrelated quality-of-life assessment for patients with multiple myeloma. Br J Haematol 1999;104:605-611.

23. Gulbrandsen N, Wisloff F, Brinch L, et al. Healthrelated quality of life in multiple myeloma patients receiving high-dose chemotherapy with autologous blood stem-cell support. Med Oncol 2001;18:65-77.

24. Wisloff F, Huorth M. Health-related quality of life assessed before and during chemotherapy predicts for survival in multiple myeloma. Nordic Myeloma Study Group. Br J Haematol 1997;97:29-37. 
Quality of life in multiple myeloma

Appendix. Additional questions concerning multiple myeloma and treatment related symptoms. Have you, during the past week, been bothered by:

\begin{tabular}{lcccc}
\hline & Not at all & A little & Quite a bit & Very much \\
\hline $\mathbf{1}$ Dizziness? & 1 & 2 & 3 & 4 \\
$\mathbf{2}$ Palpitations? & 1 & 2 & 3 & 4 \\
$\mathbf{3}$ Pain in the chest? & 1 & 2 & 3 & 4 \\
$\mathbf{4}$ Pain in the back? & 1 & 2 & 3 & 4 \\
$\mathbf{5}$ Pain in the arms? & 1 & 2 & 3 & 4 \\
$\mathbf{6}$ Pain in the legs? & 1 & 2 & 3 & 4 \\
$\mathbf{7}$ Thirst? & 1 & 2 & 3 & 4 \\
$\mathbf{8}$ Sleepiness? & 1 & 2 & 3 & 4 \\
$\mathbf{9}$ Fever? & 1 & 2 & 3 & 4 \\
$\mathbf{1 0}$ Weakness in arms/legs? & 1 & 2 & 3 & 4 \\
$\mathbf{1 1}$ Tingling of hands/feet? & 1 & 2 & 3 & 4 \\
$\mathbf{1 2}$ Numbness in arms/legs? & 1 & 2 & 3 & 4 \\
$\mathbf{1 3}$ Haematomas? & 1 & 2 & 3 & 4 \\
$\mathbf{1 4}$ Soreness of the mouth? & 1 & 2 & 3 & 4 \\
$\mathbf{1 5}$ Myalgia? & 1 & 2 & 3 & 4 \\
$\mathbf{1 6}$ Shivers? & 1 & 2 & 3 & 4 \\
$\mathbf{1 7}$ Perspiration? & 1 & 2 & 3 & 4 \\
$\mathbf{1 8}$ Change of taste? & 1 & 2 & 3 & 4 \\
$\mathbf{1 9}$ Diminished sexual interest? & 1 & 2 & 3 & 4 \\
$\mathbf{2 0}$ Headache? & 1 & 2 & 3 & 4 \\
$\mathbf{2 1}$ Loss of hair? & 1 & 2 & 3 & 4 \\
$\mathbf{2 2}$ Gum bleeding? & 1 & 2 & 3 & 4 \\
$\mathbf{2 3}$ Epistaxis? & 1 & 2 & 3 & 4 \\
$\mathbf{2 4}$ Bone pain/joint pain? & 1 & 2 & 3 & 4 \\
\hline
\end{tabular}

